\title{
We say farewell to our assistant editor
}

After seven years at Powder Diffraction, I will be leaving my position as assistant editor after this issue is published. Since 2001, my responsibilities have included administrative work and managing the editorial process. My most critical responsibility has been proofing and editing manuscripts approved for publication by the editor-in-chief. In particular, I edit and make sure text is written as clearly as possible, tables and figures are presented properly, and references are accurate and cited correctly. New data, methods, and results must be communicated clearly for scientific innovation and progress to continue. This is why careful proofing and editing of papers is critical, and it is my hope I have contributed to this in some way.

One of the major changes I implemented a few years ago was changing the way we process manuscripts. Before we started handling manuscripts electronically, submissions, reviews, revisions, and the final product were all received and sent by postal mail. Seeing that sending hard copies back and forth was time consuming and less efficient, I transitioned this journal's processing of manuscripts to an electronic method using the existing resources we had (like e-mail accounts and FTP servers). This has allowed us to edit papers directly, handle large figure files, communicate with authors and reviewers quickly, and submit papers to the publisher expeditiously without increasing our costs.

But our current handling of manuscripts electronically is still a manual method requiring lots of organization of correspondence, reviews, and content files. Although this is manageable with the current number of papers we receive every year, we hope and expect that more manuscripts in the future will be submitted and therefore published in Powder Diffraction. As a result, and also because we want to provide an even easier and automated process to our authors and staff, we announced earlier this year our intention to begin using a web-based manuscript submission and peer review system called Peer X-Press (PXP) by the American Institute of Physics (AIP). We still hope to have PXP up and running later this year.

As I sign off, I reiterate the commitment of Powder Diffraction in improving manuscripts and processing them quickly for publication. As a result, I am confident Powder Diffraction will be your journal of choice for communicating your work in materials characterization and crystallography to the world.

Allen Huang
Assistant Editor

I acknowledge Allen's exceptional service and dedicated work in maintaining the high editorial standards of Powder Diffraction. He began as an editorial assistant in 2001 and later became assistant editor. In addition to his contributions to Powder Diffraction, Allen also edited and prepared manuscripts for Advances in X-ray Analysis. He has spent countless hours on his duties, which has been a great sacrifice on his part. Without Allen, both Powder Diffraction and Advances in $X$-ray Analysis would not be the publications they are today.

Finally I sincerely thank Allen for his outstanding contributions and sacrifice over the last seven years that have benefited the powder diffraction and materials science communities as a whole. I wish Allen all the best in the future.

Ting C. Huang

Editor-in-Chief 